\title{
LA INFLUENCIA DE LAS TICS EN LA DOCENCIA UNIVERSITARIA DE LA FACULTAD DE CIENCIAS CONTABLES DE LA UNMSM
}

THE INFLUENCE OF TIC`S IN UNIVERSITY TEACHING AT THE FACULTY ACCOUNTING STUDIES OF SAN MARCOS UNIVERSITY

\author{
CPCC. Raúl Jesús Vergara Moncada*
}

Docente Auxiliar de la Facultad de Ciencias Contables, UNMSM CPCC. Adolfo Valencia Gutiérrez**

Docente Auxiliar de la Facultad de Ciencias Contables, UNMSM

[Recepción: Setiembre de 2010/ Conformidad: Setiembre de 2010]

\begin{abstract}
RESUMEN
En la actualidad, los docentes del Sistema Universitario necesitamos conocer los múltiples conceptos que se utilizan en el mundo de las Nuevas Tecnologías de Información y Comunicación (NTICs), por lo cual el presente trabajo de investigación pretende aclarar algunos conceptos relativos a dichos medios en el proceso de Enseñanza-Aprendizaje a través de medios tecnológicos como los aportes y la problemática de las TICs, las Universidades y el Uso de la Web, el estudiante universitario como elemento central en un nivel de auto Aprendizaje, la actuación del docente universitario frente a entornos virtuales y finalmente una encuesta a los Docentes de la Facultad de Ciencias Contables de la UNMSM, conclusiones y recomendaciones.

Palabras Claves: Tecnologías de Información y Comunicación, Aula Virtual, Metodología.
\end{abstract}

\begin{abstract}
At present the teachers of the University System we need to know the multiple concepts that are in use in the world of the New Technologies of Information and Communication (NTICs), for which the present work of investigation the university student tries to clarify some concepts relative to the above mentioned means in the process of Education Learning across technological means as the contributions and the problematics of the TICs, the Universities and the Use of the Web, as central element in a level of car learning, the action of the university teacher opposite to virtual environments and finally a survey to the Teachers of the Faculty of Countable Sciences of the UNMSM, conclusions and recommendations.

Key Words: Technologies of Information and Communication, Virtual Classroom, Methodology.
\end{abstract}

* Contador Público Colegiado Certificado. Docente Auxiliar de la Facultad de Ciencias Contables, UNMSM. E-mail: rvergara64@yahoo.es

** Doctorado en Ciencias de la Educación. Coordinador de la Diplomatura en Gestión Tributaria de la Unidad de Postgrado de la Facultad de Ciencias Contables, UNMSM.

E-mail: avalencia@ladersam.com 


\section{INTRODUCCIÓN}

Términos nuevos comienzan a formar parte del vocabulario, directamente relacionados con conceptos y servicios que una formación continua del docente universitario para obtener una gestión correcta y actualizada: Universidad o Campus Virtual, Videoconferencias, On-Line, Web de la Facultad, Web de la Asignatura (donde se incluyen servicios de todo tipo: el programa, información de los temas, noticias de interés, ejemplos de exámenes, recursos, e-mail; blogs, etc.

La presencia de la TICs en los procesos enseñanza y aprendizaje nos plantea una serie de interrogantes: el primero de ellos sería si estamos ante un nuevo paradigma de la enseñanza.

Si estamos conforme con esta afirmación debemos ser conscientes de nuevos contextos para el docente universitario: una nueva dimensión de su formación, una relación de competencias básicas, un uso de novedosos recursos para llevar a cabo la investigación. Pero todo esto sería solo una exposición teórica, si no se dota de infraestructura necesaria, hablamos de: servidores, intranet, aulas virtuales, salas de videoconferencias que es necesario que el actual docente universitario de contabilidad conozca.

\section{OBJETIVO GENERAL}

Determinar en qué medida las TICs influyen en la labor de los docentes universitarios.

\section{OBJETIVOS ESPECÍFICOS}

Analizar el nivel de conocimiento de las TICs por parte de los docentes de la Facultad de Ciencias Contables de la UNMSM.

Verificar la utilización del aula virtual por parte de los docentes de la Facultad de Ciencias Contables de la UNMSM.
Analizar la implementación del uso de las TICs en el desarrollo de los cursos de los docentes de la Facultad de Ciencias Contables de la UNMSM.

\section{MARCO CONCEPTUAL}

\section{Entendiendo las TICs}

Las tecnologías de la información y la comunicación (TIC) son un conjunto de técnicas, desarrollos y dispositivos avanzados que integran funcionalidades de almacenamiento, procesamiento y transmisión de datos.

Las tecnologías de la información y las comunicación forman parte de la cultura tecnológica que nos rodea y con la que debemos convivir. Amplían nuestras capacidades físicas y mentales, y las posibilidades de desarrollo social.

Incluimos en el concepto TIC no solamente la informática y sus tecnologías asociadas, telemática y multimedia, sino también los medios de comunicación de todo tipo: los medios de comunicación social (redes Sociales) y los medios de comunicación Interpersonales tradicionales con soporte tecnológico como el celular, fax, etc.

\section{LOS APORTES DE LAS TIC}

Fácil acceso a una inmensa fuente de información.

- Proceso rápido a todo tipo de datos.

- Canales de comunicación inmediata.

- Capacidad alta de almacenamiento en nuevos dispositivos.

- Automatización de trabajos.

- Interactividad.

- Digitalización de información. 


\section{PROBLEMÁTICA DE LAS TICS}

- PROBLEMAS TÉCNICOS: Incompatibilidades entre sistemas, poca velocidad para navegar por Internet y para algunas tares (reconocimiento de voz y videos).

- FALTA DE FORMACIÓN: Conocimientos teóricos y prácticos, aptitudes favorables.

- PROBLEMAS DE SEGURIDAD: Accesos no autorizados, inseguridad al realizar compras virtuales.

- BARRERAS ECONÓMICAS: A pesar del progresivo abaratamiento del precio de los equipos es aún alto para muchos y la obsolescencia exige nuevas tecnologías.

- BARRERAS CULTURALES: El idioma inglés es el predominante.

\section{LAS TICS EN EL CONTEXTO DE INTERNET}

De todos los elementos que integran las TIC, sin duda, el más poderoso y revolucionario es Internet, que nos abre las puertas de una nueva era: la Era Internet. Ella se ubica la actual sociedad de la información. Internet nos proporciona un tercer mundo en el que podemos hacer casi todo lo que hacemos en el mundo real y además nos permite desarrollar nuevas actividades, muchas de ellas enriquecedoras para nuestra personalidad y forma de vida (contactar con foros telemáticos y personas de todo el mundo, localización inmediata de cualquier tipo de información, teletrabajo, teleformación, etc. Ahora las personas podemos repartir el tiempo de nuestra vida interactuando en tres mundos: el mundo presencial, de naturaleza física, constituido por átomos, regido por las leyes del espacio, en el que hay distancias entre las cosas y las personas; el mundo intrapersonal de la imaginación y el ciberespacio, de naturaleza virtual, sin distancias.

\section{LAS UNIVERSIDADES Y EL USO DE LA WEB}

Es evidente que es necesario cambiar el modelo educativo actual y evolucionar hacia otro donde la formación universitaria reglada y de posgrado atienda adecuadamente a la demanda existente pero con calidad.

Ante estas necesidades, las tecnologías de la información y de las comunicaciones pueden ser uno de los elementos clave que ayuden a las transformaciones requeridas. Así lo han entendido muchas universidades peruanas que con distintas intensidades de uso de la Web ofertan asignaturas, cursos y carreras totalmente virtuales o en modalidad mixta.

\section{USO DE LA WEB EN LA EDUCACIÓN UNIVERSITARIA}

Es necesario cambiar el modelo educativo actual y evolucionar hacia otro donde la formación universitaria de pregrado y postgrado atienda adecuadamente a la demanda existente pero con calidad.

Claro está que las características del aprendizaje donde profesor y alumno no coinciden en el espacio y en el tiempo se diferencian sustancialmente de las correspondientes a la enseñanza presencial. Esta realidad requiere de una metodología que cambie la forma tradicional de cómo el estudiante aprende y el profesor enseña.

Esta nueva forma en que se produce el proceso de enseñanza-aprendizaje obliga a perfilar las funciones de los elementos que componen el sistema, entre los que destacan: el estudiante, el profesorado, los materiales formativos $y$ el entorno virtual de aprendizaje. 


\section{EL ESTUDIANTE UNIVERSITARIO COMO ELEMENTO CENTRAL EN UN NIVEL DE AUTOAPRENDIZAJE}

El elemento básico en todo proceso de enseñanza-aprendizaje debe ser el destinatario del mismo y en función del que se diseña todo el proceso: el aprendiz. En este sentido, todos los elementos que componen el sistema deben ponerse a su disposición para que pueda gestionar su proceso de formación.

La actividad del estudiante, entendida como el conjunto de acciones y operaciones ha de ser parte vital del proceso de aprendizaje, el resultado de este aprendizaje es fruto de esta actividad directa y personal.

\section{ACTUACIÓN DEL DOCENTE UNIVERSITARIO FRENTE A ENTORNOS VIRTUALES}

El hecho de asumir que la relación entre profesores y estudiantes está condicionada por el entorno donde ésta se produce, en la formación presencial la relación es sincrónica mientras que la que se produce en entornos virtuales es asincrónica, permite entender claramente que las estrategias pedagógicas deben ser distintas en cada caso. Por lo tanto, no se trata de que el profesor actúe en entornos virtuales con las mismas coordenadas que realizaba en la enseñanza presencial, ni siquiera adaptar lo que en el otro entorno ejecutaba. Hay que hacerse cargo de que se interviene en un sistema distinto, donde los estudiantes, los materiales, el entorno y el profesor actúan de acuerdo con la nueva realidad.

\section{LA LABOR TUTORIAL DEL DOCENTE UNIVERSITARIO}

Las tareas de la acción tutorial por parte del docente universitario en la formación on line son distintos a la práctica habitual en la for- mación presencial donde la tutoría se realiza a demanda del estudiante.

En ellas las tareas son:

- Presentación a los estudiantes del curso y de las normas de funcionamiento.

- Resolver de forma individual y colectiva las diferentes dudas que vayan surgiendo de interacción con los materiales.

- Fomentar la participación de los estudiantes.

- Fomentar la participación de todos en actividades de trabajo cooperativo.

- Realizar las valoraciones de las actividades realizadas.

- Utilizar esa información para reorientar el aprendizaje de los estudiantes que lo necesiten.

- Desarrollar una evaluación continua formativa.

- Asesorar en métodos de estudio en la red.

- Facilitar información adicional para la aclaración y profundización en conceptos.

\section{LOS MATERIALES DIDÁCTICOS EN LA WEB}

Los materiales didácticos son el eje sobre el que se desarrollará el curso. Sobre ellos se basará la actuación del profesorado y la evaluación. Decir que los materiales didácticos para el aprendizaje on line deben estar diseñados de acuerdo con el perfil del estudiante y con los objetivos de aprendizaje planteados es obvio, pero quizá no lo sea tanto decir que los materiales didácticos deben diseñarse en armonía con el modelo de formación donde están inmersos.

Los materiales didácticos, por una parte, deberán potenciar la interacción entre profesores y estudiantes y, por otra, utilizarán adecuadamente las prestaciones del entorno de formación, es decir, combinarán el texto, las ilustraciones, las simulaciones y el video. 


\section{LA EVALUACIÓN CONTINUA}

La evaluación continua debe entenderse como un instrumento para favorecer la consecución de los objetivos propuestos en un curso. En este sentido, no se diferencia de otras actividades de aprendizaje propuestas cuyo fin es asegurar un aprendizaje eficaz. Sin embargo, el valor añadido de una prueba al final de cada tema permite una cierta calendarización del curso.

\section{LA GUÍA PARA APRENDER}

La guía de aprendizaje para el estudiante pretende facilitar su labor activa de autoaprendizaje proporcionándole toda la información que necesita conocer al principio del curso: objetivos, metodología de trabajo, temario, criterios de evaluación, calendarización y bibliografía.

\section{EL ENTORNO VIRTUAL PARA APREN- DER}

Las funciones que tiene el aula en la enseñanza presencial son asumidas por los entornos virtuales cuando el aprendizaje se desarrolla sin coincidir en el espacio ni en el tiempo.

Entre sus características destacan:

- Permite la interacción de los estudiantes con sus compañeros y con los profesores.

- Permite la interactividad de los estudiantes con los materiales.

Entre las reflexiones que el entorno debe suscitar merecen especial atención:

- El entorno tecnológico es un medio y no un fin en sí mismo

- El entorno tecnológico puede condicionar, y condiciona el modelo pedagógico.
RESULTADO DE LA ENCUESTA

APLICADA A PROFESORES DE

LA FACULTAD DE CIENCIAS

CONTABLES DE LA UNMSM

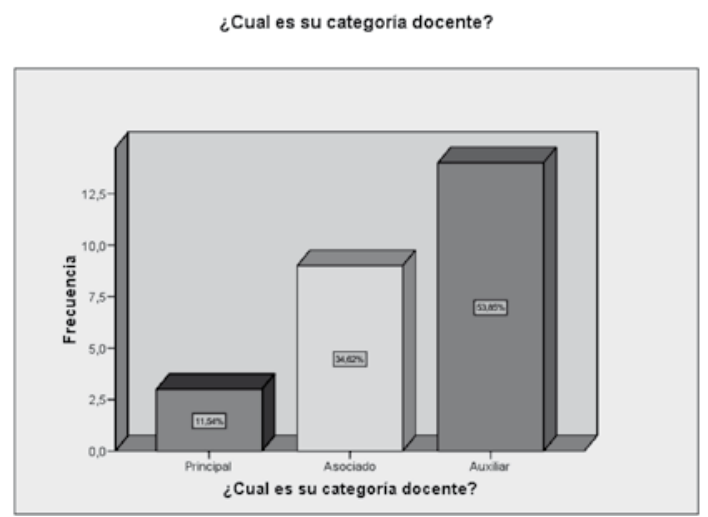

Fuente: F.C.C. UNMSM

Elaboración Propia

¿El curso que Ud. enseña se encuentra dentro de?

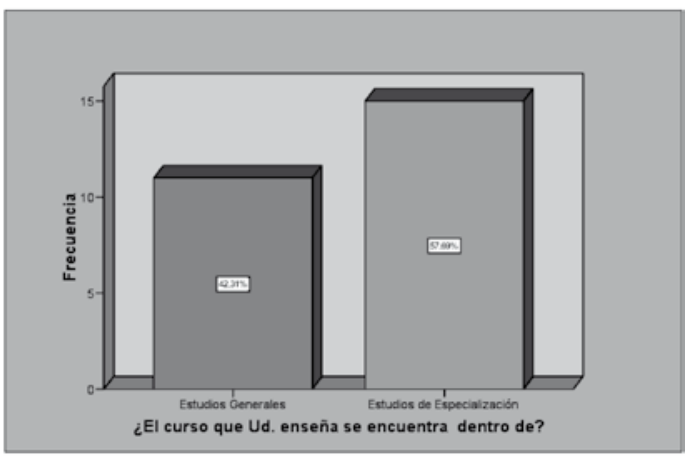

Fuente: F.C.C. UNMSM.

Elaboración propia.

¿Cuenta Ud. con computadora?

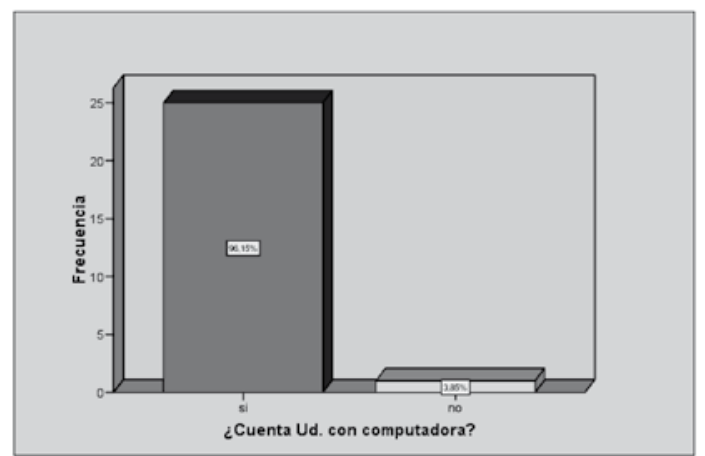

Fuente: F.C.C. UNMSM.

Elaboración propia. 
¿Cuenta Ud. con aceso a internet

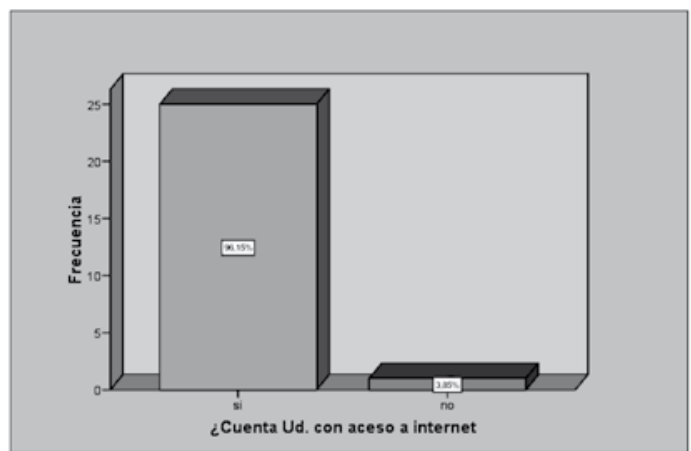

Fuente: F.C.C. UNMSM.

Elaboración propia.

¿Desde donde accede al servicio de internet?

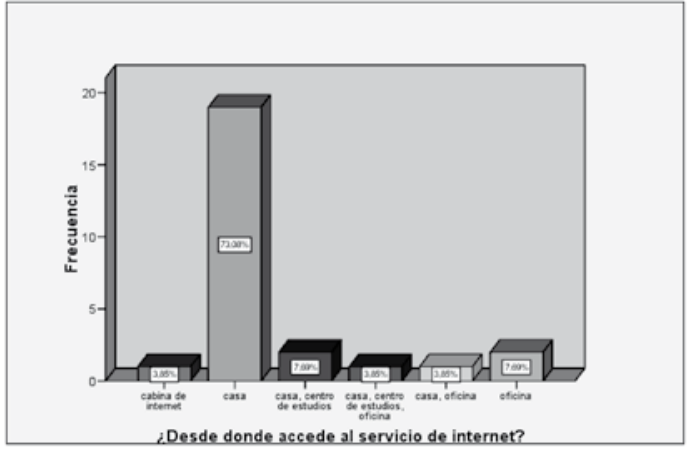

Fuente: F.C.C. UNMSM.

Elaboración propia.

¿Cuenta con correo electrónico?

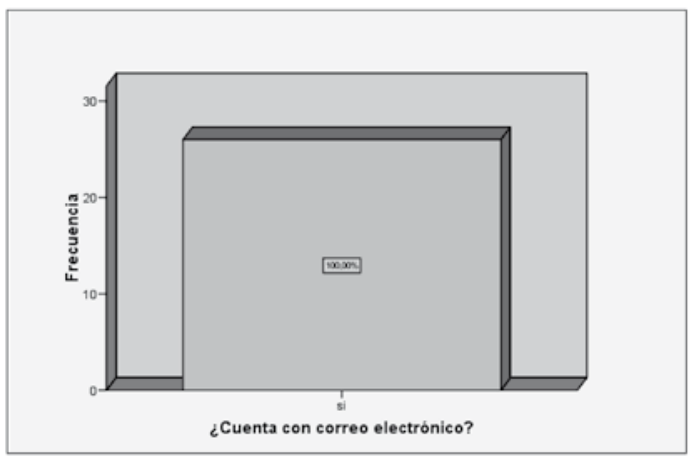

Fuente: F.C.C. UNMSM.

Elaboración propia.
¿Con que frecuencia accede a su cuenta de correo electrónico?

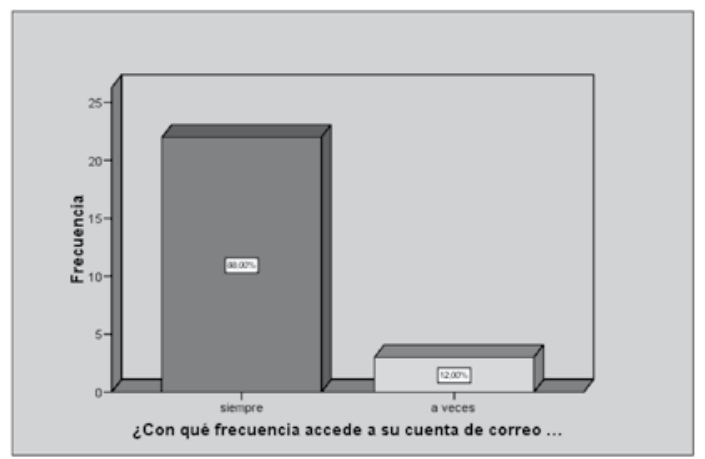

Fuente: F.C.C. UNMSM.

Elaboración propia.

¿Que programas informaticos domina?

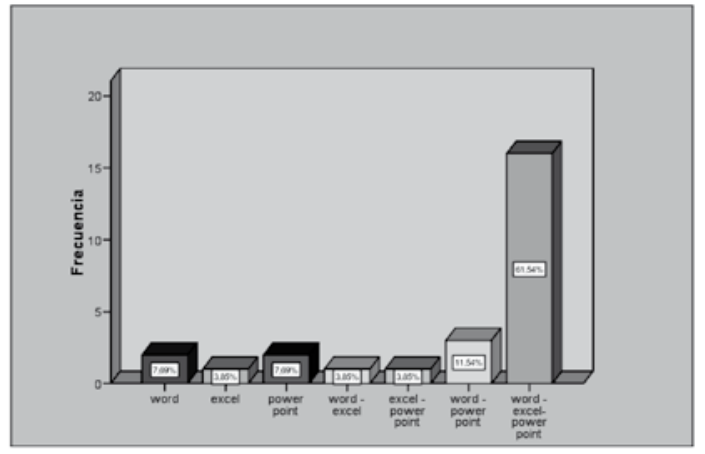

Fuente: F.C.C. UNMSM.

Elaboración propia.

¿Qué programas informáticos utiliza para la elaboración de sus cursos?

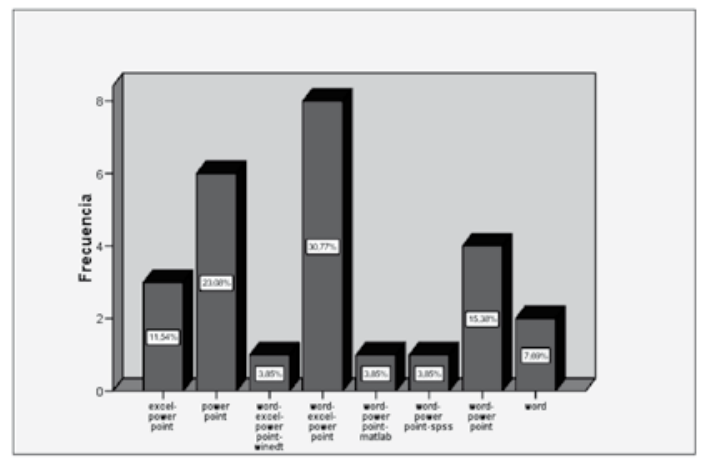

Fuente: F.C.C. UNMSM.

Elaboración propia. 
¿Cuenta con recursos informaticos en la web?

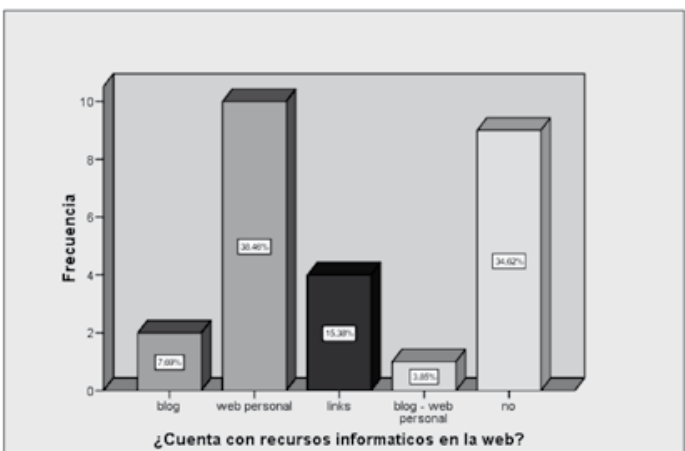

Fuente: F.C.C. UNMSM.

Elaboración propia.

¿Dentro de la metodologia de su curso recomienda a sus alumnos enlaces a

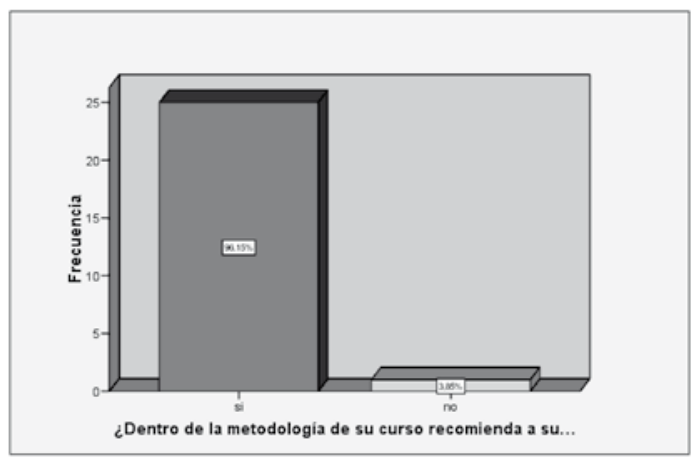

Fuente: F.C.C. UNMSM.

Elaboración propia.

¿Considera Ud. importante el uso de las TICs en el desarrollodel curso?

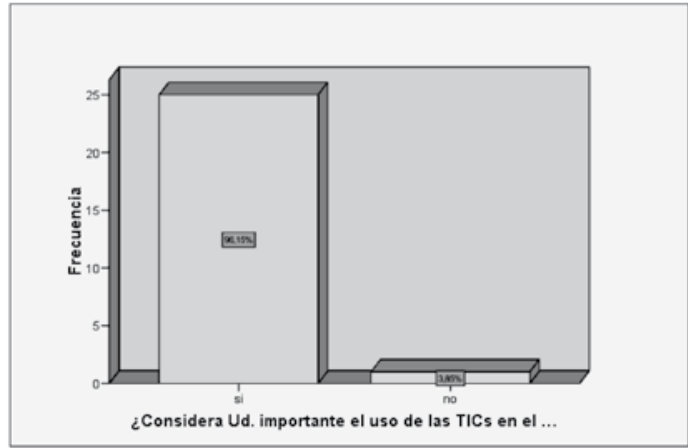

Fuente: F.C.C. UNMSM.

Elaboración propia.

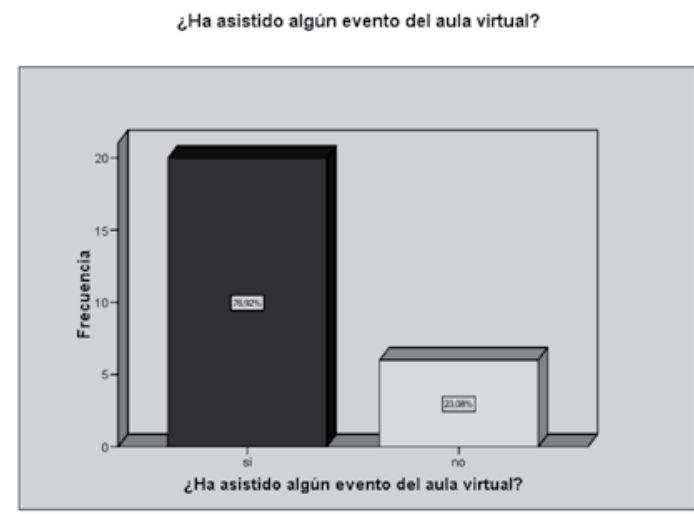

Fuente: F.C.C. UNMSM

Elaboración propia.

¿Conoce Ud. de la existencia del aula virtual de la FCC.UNMSM?

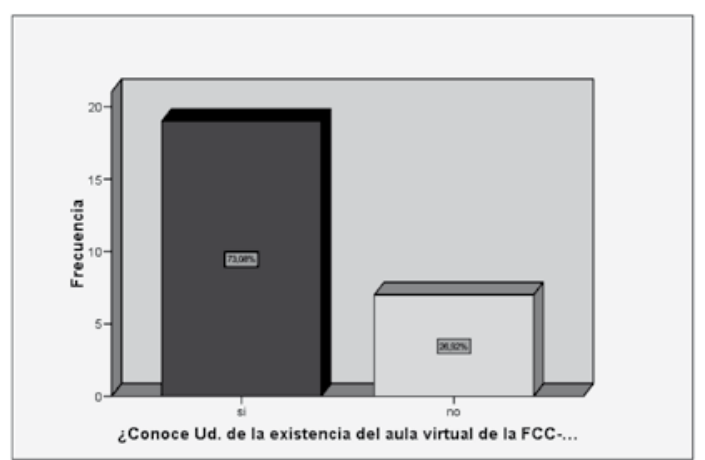

Fuente: F.C.C. UNMSM

Elaboración propia.

¿Utiliza Ud. el aula virtual de la FCC-UNMSM?

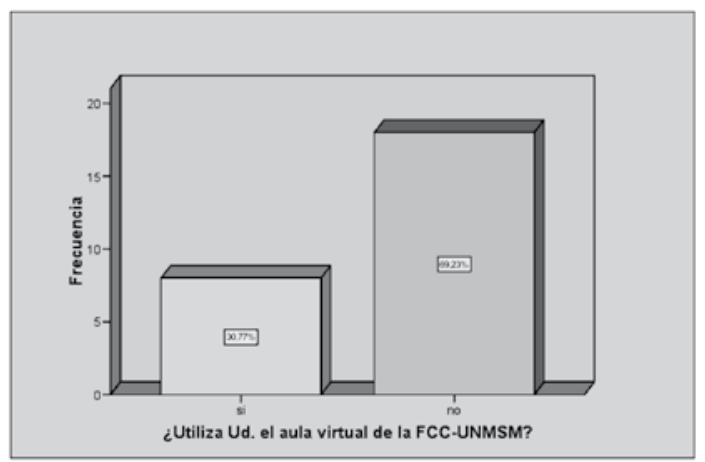

Fuente: F.C.C. UNMSM.

Elaboración propia. 


\section{CONCLUSIONES}

1. Las TICs inciden en los procesos de enseñanza-aprendizaje en la Facultad de Ciencias Contables de la UNMSM.

2. Falta más implementación de recursos tecnológicos.

3. Insuficiente Asistencia Técnica para la Implementación del aula virtual para los docentes.

4. Ausencia de sensibilización docente en temas de tecnología de información y Comunicación.

\section{RECOMENDACIONES}

1.- Que progresivamente más docentes utilicen las TICs en el desarrollo de sus cursos (aula virtual).

2.- Que se implementen cada vez más recursos tecnológicos y se gestione la adquisición por parte de la F.C.C. una laptop con descuento por Planilla.

3.- Se brinde asistencia técnica a través de la Unidad de Tecnología Educativa de la F.C.C. UNMSM.

4.- Lograr la sensibilización en temas de tecnologías de información y comunicación de los docentes, con capacitación en seminarios, talleres, cursos, etc.

\section{REFERENCIAS BIBLIOGRÁFICAS}

1. Quipukamayoc - UNMSM. Primer Semestre 2004. 2004 pág. 73.

2. Introducción a los Sistemas de Base de Datos Date C.H. 2001 Págs. 3-15.
3. Diseño de Base de Datos. Miguel Adoración, Martínez Paloma. 2000 Pág. 2

4. Rondinel Sosa, Rocio. La regularización de las Nuevas Tecnología en la Administración Pública en el Perú.

5. Funciones de la Oficina Nacional de Gobierno Electrónico e Informática D.S.067-2003-PCM

6. Bricall, J.M. La Universidad, al final del milenio (1996)

Web Site:

- Vilaseca, J. (2001): “Tecnologías de la información y la comunicación en las universidades"[documento electrónico] http://www.uoc.es/web/esp/articles/ vilaseca/tic_imp.html

- Martínez, Javier (2004). El papel del tutor en el aprendizaje virtual [artículo en línea].UOC. [Fecha de consulta: 17/01/05].

http://www.uoc.edu/dt/20383/index.html

- Escudero, Tomás, 1999, Evaluación de los aprendizajes en la universidad http://www.ub.edu/forum/escudero.htm.

- Escudero, Tomás, 2002, "Indicadores: Información estratégica para el Sistema universitario español" en CRUE, La Universidad Española en cifras (2002) http://www.crue.org/cdOBSERVATO$\mathrm{RIO} /$ index.htm

- Adell, J. (1997): Tendencias en educación en la sociedad de las tecnologías de la información. EDUTEC. Revista Electrónica de Tecnología Educativa, N. ${ }^{\circ}$ 7 http://www.uib.es/depart/gte/revelec7.html 